\title{
Refinements and Generalizations of Functional Dresher's and Bellman's Inequalities
}

\author{
Rabia Bibi and Muhammad Shahzad \\ Department of Mathematics, Hazara University, Mansehra 21300, Pakistan \\ Correspondence should be addressed to Rabia Bibi; emaorr@gmail.com \\ Received 11 May 2015; Accepted 6 July 2015 \\ Academic Editor: Changbum Chun
}

Copyright (C) 2015 R. Bibi and M. Shahzad. This is an open access article distributed under the Creative Commons Attribution License, which permits unrestricted use, distribution, and reproduction in any medium, provided the original work is properly cited.

We give refinements and generalizations of the Dresher and Bellman inequalities for positive linear functionals. We also give reverse of the new obtained generalized version of these inequalities. Finally, we apply our results on time scales integrals to obtain refinements and generalizations of time scales Dresher's and Bellman's inequalities.

\section{Introduction}

Dresher's and Bellman's inequalities are obtained from the classical Hölder and Minkowski inequalities and are well known in the theory of inequalities and means. For introduction and some applications of these classical inequalities, we refer to [1-5]. Due to the importance of Dresher's and Bellman's inequalities, there are various generalizations, refinements, and variants that appeared in the literature. In this paper, we give refinements and further generalizations of the generalized version of these inequalities for positive linear functionals and time scales integrals. For this, first we recall the definition of positive linear functionals and the two functional inequalities from [5].

Definition 1 (positive linear functional). Let $E$ be a nonempty set and let $L$ be a linear class of real valued functions $f: E \rightarrow$ $\mathbb{R}$ having the following properties:

$$
\begin{aligned}
& \left(\mathrm{L}_{1}\right) \text { If } f, g \in L \text { and } a, b \in \mathbb{R} \text {, then }(a f+b g) \in L . \\
& \left(\mathrm{L}_{2}\right) \text { If } f(t)=1 \text { for all } t \in E \text {, then } f \in L .
\end{aligned}
$$

An isotonic positive linear functional is a functional $A: L \rightarrow$ $\mathbb{R}$ having the following properties:

$$
\begin{aligned}
& \left(\mathrm{A}_{1}\right) \text { If } f, g \in L \text { and } a, b \in \mathbb{R} \text {, then } A(a f+b g)=a A(f)+ \\
& b A(g) \text {. } \\
& \left(\mathrm{A}_{2}\right) \text { If } f \in L \text { and } f(t) \geq 0 \text { for all } t \in E \text {, then } A(f) \geq 0 .
\end{aligned}
$$

Remark 2. Sums and Lebesgue integrals are the most familiar examples of positive linear functionals. In [6] it is shown that time scales integrals including the Cauchy, Riemann, Lebesgue, multiple Riemann, multiple Lebesgue delta, nabla, and diamond- $\alpha$ also satisfy the properties of positive linear functionals.

Theorem 3 (Dresher's inequality [5, Theorem 4.21]). Let $E$ and $L$ be such that $\left(L_{1}\right)$ and $\left(L_{2}\right)$ are satisfied and suppose that both $A$ and $B$ satisfy $\left(A_{1}\right)$ and $\left(A_{2}\right)$. If $f_{i}, g_{i}: E \mapsto[0, \infty)$ with

$$
f_{i}^{p},\left(\sum_{i=1}^{n} f_{i}\right)^{p}, g_{i}^{r},\left(\sum_{i=1}^{n} g_{i}\right)^{r} \in L,
$$

where $p \geq 1>r>0$ and $B\left(g_{i}^{r}\right)>0$ for $1 \leq i \leq n$, then

$$
\left(\frac{A\left(\left(\sum_{i=1}^{n} f_{i}\right)^{p}\right)}{B\left(\left(\sum_{i=1}^{n} g_{i}\right)^{r}\right)}\right)^{1 /(p-r)} \leq \sum_{i=1}^{n}\left(\frac{A\left(f_{i}^{p}\right)}{B\left(g_{i}^{r}\right)}\right)^{1 /(p-r)} .
$$

Theorem 4 (Bellman's inequality [5, Theorem 4.29]). Let $E, L$, and $A$ be such that $\left(L_{1}\right),\left(L_{2}\right),\left(A_{1}\right)$, and $\left(A_{2}\right)$ are satisfied. For $p>1$, assume that $f, g: E \mapsto[0, \infty)$ are such that $f^{p}, g^{p},(f+$ $g)^{p} \in L$. Suppose that $f_{0}, g_{0}>0$ are such that

$$
\begin{gathered}
f_{0}^{p}-A\left(f^{p}\right)>0, \\
g_{0}^{p}-A\left(g^{p}\right)>0 .
\end{gathered}
$$


Then the following inequality holds:

$$
\begin{aligned}
& \left(\left(f_{0}^{p}-A\left(f^{p}\right)\right)^{1 / p}+\left(g_{0}^{p}-A\left(g^{p}\right)\right)^{1 / p}\right)^{p} \\
& \quad \leq\left(f_{0}+g_{0}\right)^{p}-A\left((f+g)^{p}\right) .
\end{aligned}
$$

Remark 5. The inequality in Theorem 4 also holds if $p<0$. Moreover, it holds in reverse order if $0<p<1$.

Recently, the author, Rabia Bibi, gives the following refinement of Bellman's inequality.

Theorem 6 (refinement of Bellman's inequality [7, Theorem 2.1]). Let $L$ satisfy conditions $\left(L_{1}\right)$ and $\left(L_{2}\right)$ and let $A$ satisfy conditions $\left(A_{1}\right)$ and $\left(A_{2}\right)$ on a nonempty set E. For $p \geq 2$, assume that $f, g$ are nonnegative functions on $E$ such that $(f+g)^{p}, f^{p}, g^{p} \in L$ and $A\left((f+g)^{p}\right)>0$. Suppose that $f_{0}, g_{0}>0$ are such that

$$
\begin{aligned}
& f_{0}^{p}-A\left(f^{p}\right)>0, \\
& g_{0}^{p}-A\left(g^{p}\right)>0 .
\end{aligned}
$$

Then the following inequality holds:

$$
\begin{aligned}
& \left(\left(f_{0}^{p}-A\left(f^{p}\right)\right)^{1 / p}+\left(g_{0}^{p}-A\left(g^{p}\right)\right)^{1 / p}\right){ }^{p} \leq\left[\left(f_{0}^{p}\right.\right. \\
& \left.-A\left(\left|f-(f+g) \frac{A\left(f(f+g)^{p-1}\right)}{A\left((f+g)^{p}\right)}\right|^{p}\right)\right)^{1 / p} \\
& +\left(g g_{0}^{p}\right. \\
& \left.\left.-A\left(\left|g-(f+g) \frac{A\left(g(f+g)^{p-1}\right)}{A\left((f+g)^{p}\right)}\right|^{p}\right)\right)^{1 / p}\right]^{p} \\
& -A\left((f+g)^{p}\right) .
\end{aligned}
$$

In Section 2, we give refinements of Dresher's inequality for positive linear functionals (Theorem 3). In Section 3, we generalize Dresher's inequality and new refinements obtained in Section 2. Further, we obtain the reverse of new generalized Dresher's inequality. Section 4 provides the generalizations of Bellman's inequality and its refinement (Theorem 18) and reverse of the new generalized Bellman's inequality. Finally, in Section 5, we apply the obtained results to time scales integrals and obtain improvements of Dresher's and Bellman's inequalities on time scales.

In order to prove our main results, we use the classical Hölder and Minkowski inequalities, and the following refinements of them (see [8]).

Theorem 7 (see [8, Theorem 13]). Let L satisfy conditions $\left(L_{1}\right)$ and $\left(L_{2}\right)$ and let $A$ satisfy conditions $\left(A_{1}\right)$ and $\left(A_{2}\right)$ on a nonempty set $E$. Let $p \geq 2$ and define $q$ by $1 / p+1 / q=1$. Then, for all nonnegative functions $w, g, h \in L$ such that

$$
\begin{array}{r}
w g h, w g^{p}, w h^{q}, w\left|g-h^{q-1} \frac{A(w g h)}{A\left(w h^{q}\right)}\right|^{p} \in L, \\
A\left(w h^{q}\right)>0,
\end{array}
$$

the inequality

$$
\begin{aligned}
& A(w g h) \\
& \quad \leq\left[A\left(w g^{p}\right)-A\left(w\left|g-h^{q-1} \frac{A(w g h)}{A\left(w h^{q}\right)}\right|^{p}\right)\right]^{1 / p} \\
& \cdot A^{1 / q}\left(w h^{q}\right)
\end{aligned}
$$

holds. In the case $0<p<2$, inequality in (8) is reversed.

Theorem 8 (see [8, Theorem 14]). Let $L$ and $A$ be as in Theorem 7. If $p \geq 2$, then for all nonnegative functions $w, g, h$ on $E$ such that $w(g+h)^{p}, w g^{p}, w h^{p} \in L$, and $A\left(w(g+h)^{p}\right)>0$, the inequality

$$
\begin{aligned}
& A^{1 / p}\left(w(g+h)^{p}\right) \leq\left(A\left(w g^{p}\right)\right. \\
& \left.-A\left(w\left|g-(g+h) \frac{A\left(w g(g+h)^{p-1}\right)}{A\left(w(g+h)^{p}\right)}\right|^{p}\right)\right)^{1 / p} \\
& +\left(A\left(w h^{p}\right)\right. \\
& \left.-A\left(w\left|h-(g+h) \frac{A\left(w h(g+h)^{p-1}\right)}{A\left(w(g+h)^{p}\right)}\right|^{p}\right)\right)^{1 / p}
\end{aligned}
$$

holds.

Remark 9. From the proof of Theorem 8 (see [8]), it is obvious that inequality (9) holds in reverse order for $0<p<1$ or $1<p<2$.

We also need the following classical Radon's inequality in order to prove the reverse of Dresher's inequality.

Theorem 10 (see [1, Theorem 3, page 181]). Let $a_{i} \geq 0$ and let $b_{i}>0$ for $i=1, \ldots$, n. If $p>1$, then

$$
\frac{\left(\sum_{i=1}^{n} a_{i}\right)^{p}}{\left(\sum_{i=1}^{n} b_{i}\right)^{p-1}} \leq \sum_{i=1}^{n} \frac{a_{i}^{p}}{b_{i}^{p-1}} .
$$

If $p<1$, then inequality (10) holds in reverse order.

\section{Refinements of Dresher's Inequality}

Our first result gives the refinement of Theorem 3 , for $p>2$. 
Theorem 11. Let $E$ and $L$ be such that $\left(L_{1}\right)$ and $\left(L_{2}\right)$ are satisfied and suppose that both $A$ and $B$ satisfy $\left(A_{1}\right)$ and $\left(A_{2}\right)$. Suppose that $w, f_{i}, g_{i}: E \mapsto[0, \infty)$ are such that

$$
w f_{i}^{p}, w\left(\sum_{i=1}^{n} f_{i}\right)^{p}, w g_{i}^{r}, w\left(\sum_{i=1}^{n} g_{i}\right)^{r} \in L,
$$

where $p>2,1>r>0$ such that $p \geq 2(p-r)$ and $B\left(w g_{i}^{r}\right)>0$ for $1 \leq i \leq n$. Then

$$
\begin{aligned}
& {\left[\frac{A\left(w\left(\sum_{i=1}^{n} f_{i}\right)^{p}\right)}{B\left(w\left(\sum_{i=1}^{n} g_{i}\right)^{r}\right)}\right]^{1 /(p-r)}} \\
& \quad \leq \sum_{i=1}^{n}\left[\frac{A\left(w f_{i}^{p}\right)}{B\left(w g_{i}^{r}\right)}\right]^{1 /(p-r)}-K
\end{aligned}
$$

holds, where

$$
\begin{aligned}
K & =\sum_{i=1}^{n} \mid\left(\frac{A\left(w f_{i}^{p}\right)}{B\left(w g_{i}^{r}\right)}\right)^{1 / p} \\
& -\left.B^{(p-r) / r p}\left(w g_{i}^{r}\right) \frac{\sum_{i=1}^{n} A^{1 / p}\left(w f_{i}^{p}\right)}{\sum_{i=1}^{n} B^{1 / r}\left(w g_{i}^{r}\right)}\right|^{p /(p-r)}
\end{aligned}
$$

Proof. By using the Minkowski inequality for positive linear functionals, we get

$$
\begin{gathered}
A^{1 / p}\left(w\left(\sum_{i=1}^{n} f_{i}\right)^{p}\right) \leq \sum_{i=1}^{n} A^{1 / p}\left(w f_{i}^{p}\right), \\
B^{1 / r}\left(w\left(\sum_{i=1}^{n} g_{i}\right)^{r}\right) \geq \sum_{i=1}^{n} B^{1 / r}\left(w g_{i}^{r}\right) .
\end{gathered}
$$

By using the discrete case of Theorem 7, we get

$$
\begin{aligned}
& \sum_{i=1}^{n} A^{1 / p}\left(w f_{i}^{p}\right)=\sum_{i=1}^{n}\left(\frac{A\left(w f_{i}^{p}\right)}{B\left(w g_{i}^{r}\right)}\right)^{1 / p} B^{1 / p}\left(w g_{i}^{r}\right) \\
& \leq\left[\sum_{i=1}^{n}\left[\frac{A\left(w f_{i}^{p}\right)}{B\left(w g_{i}^{r}\right)}\right]^{1 /(p-r)}-\sum_{i=1}^{n} \mid\left(\frac{A\left(w f_{i}^{p}\right)}{B\left(w g_{i}^{r}\right)}\right)^{1 / p}\right. \\
& \left.-\left.B^{(p-r) / r p}\left(w g_{i}^{r}\right) \frac{\sum_{i=1}^{n} A^{1 / p}\left(w f_{i}^{p}\right)}{\sum_{i=1}^{n} B^{1 / r}\left(w g_{i}^{r}\right)}\right|^{p /(p-r)}\right]^{(p-r) / p} \\
& \cdot\left(\sum_{i=1}^{n} B^{1 / r}\left(w g_{i}^{r}\right)\right)^{r / p} \cdot
\end{aligned}
$$

Now by combining (14) and (15), we obtain inequality (12).

In next theorem, we give a new inequality of the Dresher type for positive linear functionals.

Theorem 12. Let $E$ and $L$ be such that $\left(L_{1}\right)$ and $\left(L_{2}\right)$ are satisfied and suppose that both $A$ and $B$ satisfy $\left(A_{1}\right)$ and $\left(A_{2}\right)$. Suppose that $w, f_{i}, g_{i}: E \mapsto[0, \infty)$ are such that

$$
\begin{gathered}
w f_{i}^{p}, w\left(\sum_{i=1}^{n} f_{i}\right)^{p}, w f_{i}\left(\sum_{i=1}^{n} f_{i}\right)^{p-1}, \\
w g_{i}^{r}, w\left(\sum_{i=1}^{n} g_{i}\right)^{r}, w g_{i}\left(\sum_{i=1}^{n} g_{i}\right)^{r-1} \in L,
\end{gathered}
$$

where $p \geq 2,1>r>0$ or $1<r<2$ and $B\left(w g_{i}^{r}\right)>0$ for $1 \leq i \leq n$. Then

$$
\left[\frac{A\left(w\left(\sum_{i=1}^{n} f_{i}\right)^{p}\right)}{B\left(w\left(\sum_{i=1}^{n} g_{i}\right)^{r}\right)}\right]^{1 /(p-r)} \leq \sum_{i=1}^{n}\left[\frac{A\left(w f_{i}^{p}\right)-A\left(w\left|f_{i}-\sum_{i=1}^{n} f_{i}\left(A\left(w f_{i}\left(\sum_{i=1}^{n} f_{i}\right)^{p-1}\right) / A\left(w\left(\sum_{i=1}^{n} f_{i}\right)^{p}\right)\right)\right|^{p}\right)}{B\left(w g_{i}^{r}\right)-B\left(w\left|g_{i}-\sum_{i=1}^{n} g_{i}\left(B\left(w g_{i}\left(\sum_{i=1}^{n} g_{i}\right)^{r-1}\right) / B\left(w\left(\sum_{i=1}^{n} g_{i}\right)^{r}\right)\right)\right|^{r}\right)}\right]^{1 /(p-r)}
$$

holds, provided that the denominator of the right-hand side is positive.

Proof. By using Theorem 8, we get

$$
\begin{aligned}
& A^{1 / p}\left(w\left(\sum_{i=1}^{n} f_{i}\right)^{p}\right) \leq \sum_{i=1}^{n}\left(A\left(w f_{i}^{p}\right)\right. \\
& \left.\quad-A\left(w\left|f_{i}-\sum_{i=1}^{n} f_{i} \frac{A\left(w f_{i}\left(\sum_{i=1}^{n} f_{i}\right)^{p-1}\right)}{A\left(w\left(\sum_{i=1}^{n} f_{i}\right)^{p}\right)}\right|^{p}\right)\right)^{1 / p},
\end{aligned}
$$

$$
B^{1 / r}\left(w\left(\sum_{i=1}^{n} g_{i}\right)^{r}\right) \geq \sum_{i=1}^{n}\left(B\left(w g_{i}^{r}\right)\right.
$$

$$
\left.-B\left(w\left|g_{i}-\sum_{i=1}^{n} g_{i} \frac{B\left(w g_{i}\left(\sum_{i=1}^{n} g_{i}\right)^{r-1}\right)}{B\left(w\left(\sum_{i=1}^{n} g_{i}\right)^{r}\right)}\right|^{r}\right)\right)^{1 / r} .
$$

By using discrete Hölder's inequality, we get 


$$
\begin{aligned}
\sum_{i=1}^{n} & \left(A\left(w f_{i}^{p}\right)-A\left(w\left|f_{i}-\sum_{i=1}^{n} f_{i} \frac{A\left(w f_{i}\left(\sum_{i=1}^{n} f_{i}\right)^{p-1}\right)}{A\left(w\left(\sum_{i=1}^{n} f_{i}\right)^{p}\right)}\right|^{p}\right)\right)^{1 / p} \\
& =\sum_{i=1}^{n}\left(\frac{A\left(w f_{i}^{p}\right)-A\left(w\left|f_{i}-\sum_{i=1}^{n} f_{i}\left(A\left(w f_{i}\left(\sum_{i=1}^{n} f_{i}\right)^{p-1}\right) / A\left(w\left(\sum_{i=1}^{n} f_{i}\right)^{p}\right)\right)\right|^{p}\right)}{B\left(w g_{i}^{r}\right)-B\left(w\left|g_{i}-\sum_{i=1}^{n} g_{i}\left(B\left(w g_{i}\left(\sum_{i=1}^{n} g_{i}\right)^{r-1}\right) / B\left(w\left(\sum_{i=1}^{n} g_{i}\right)^{r}\right)\right)\right|^{r}\right)}\right) \\
& \cdot\left(B\left(w g_{i}^{r}\right)-B\left(w\left|g_{i}-\sum_{i=1}^{n} g_{i} \frac{B\left(w g_{i}\left(\sum_{i=1}^{n} g_{i}\right)^{r-1}\right)}{B\left(w\left(\sum_{i=1}^{n} g_{i}\right)^{r}\right)}\right|^{r}\right)\right)^{1 / p} \\
\leq & {\left[\sum_{i=1}^{n}\left[\frac{A\left(w f_{i}^{p}\right)-A\left(w\left|f_{i}-\sum_{i=1}^{n} f_{i}\left(A\left(w f_{i}\left(\sum_{i=1}^{n} f_{i}\right)^{p-1}\right) / A\left(w\left(\sum_{i=1}^{n} f_{i}\right)^{p}\right)\right)\right|^{p}\right)}{B\left(w g_{i}^{r}\right)-B\left(w\left|g_{i}-\sum_{i=1}^{n} g_{i}\left(B\left(w g_{i}\left(\sum_{i=1}^{n} g_{i}\right)^{r-1}\right) / B\left(w\left(\sum_{i=1}^{n} g_{i}\right)^{r}\right)\right)\right|^{r}\right)}\right]^{1 /(p-r)}\right]^{(p-r) / p} } \\
\times & \left(\sum_{i=1}^{n}\left(B\left(w g_{i}^{r}\right)-B\left(w\left|g_{i}-\sum_{i=1}^{n} g_{i} \frac{B\left(w g_{i}\left(\sum_{i=1}^{n} g_{i}\right)^{r-1}\right)}{B\left(w\left(\sum_{i=1}^{n} g_{i}\right)^{r}\right)}\right|^{r}\right)\right)^{1 / r}\right)^{r / p} \cdot
\end{aligned}
$$

Now by combining (18) and (19), we obtain inequality (17).

\section{Generalizations of Dresher's Inequality}

Let $W_{l}\left(x_{1}, x_{2}, \ldots, x_{l}\right), F_{m}\left(x_{1}, x_{2}, \ldots, x_{m}\right), G_{k}\left(x_{1}, x_{2}, \ldots, x_{k}\right)$, $\mathscr{F}_{n}\left(x_{1}, x_{2}, \ldots, x_{n}\right)$, and $\mathscr{G}_{t}\left(x_{1}, x_{2}, \ldots, x_{t}\right)$ be real valued functions of $l, m, k, n$, and $t$ variables, respectively. Then, throughout this section, we use the following notations:

$$
\begin{aligned}
& W_{l}=W_{l}(s)=W_{l}\left(w_{1}(s), w_{2}(s), \ldots, w_{l}(s)\right), \\
& F_{m}=F_{m}(s)=F_{m}\left(f_{1}(s), f_{2}(s), \ldots, f_{m}(s)\right), \\
& G_{k}=G_{k}(s)=G_{k}\left(g_{1}(s), g_{2}(s), \ldots, g_{k}(s)\right), \\
& \mathscr{F}_{n}=\mathscr{F}_{n}(s)=\mathscr{F}_{n}\left(\mathfrak{F}_{1}(s), \mathfrak{F}_{2}(s), \ldots, \mathfrak{F}_{n}(s)\right), \\
& \mathscr{G}_{t}=\mathscr{G}_{t}(s)=\mathscr{G}_{t}\left(\mathfrak{G}_{1}(s), \mathfrak{G}_{2}(s), \ldots, \mathfrak{G}_{t}(s)\right),
\end{aligned}
$$

where $\left\{w_{i}(s)\right\}_{i=1}^{l}, \quad\left\{f_{i}(s)\right\}_{i=1}^{m}, \quad\left\{g_{i}(s)\right\}_{i=1}^{k}, \quad\left\{\mathfrak{F}_{i}(s)\right\}_{i=1}^{n}, \quad$ and $\left\{\mathfrak{G}_{i}(s)\right\}_{i=1}^{t}$ are real valued functions defined on $E$.

The following results present the generalizations of Theorems 3,11 , and 12 , respectively.

Theorem 13. Let $W_{l}, F_{m}, G_{k}, \mathscr{F}_{n}, \mathscr{G}_{t}$ be defined as in (20) such that $W_{l}>0$ and $F_{m}, G_{k}, \mathscr{F}_{n}, \mathscr{G}_{t} \geq 0$. Suppose that $A$ and $B$ satisfy $\left(A_{1}\right)$ and $\left(A_{2}\right)$ and

$$
\begin{gathered}
W_{l} F_{m}^{p}, W_{l} G_{k}^{p}, W_{l}\left(F_{m}+G_{k}\right)^{p}, W_{l} \mathscr{F}_{n}^{r}, W_{l} \mathscr{G}_{t}^{r}, \\
W_{l}\left(\mathscr{F}_{n}+\mathscr{G}_{t}\right)^{r} \in L,
\end{gathered}
$$

where $p \geq 1>r>0$ and $B\left(W_{l} \mathscr{F}_{n}^{r}\right), B\left(W_{l} \mathscr{G}_{t}^{r}\right)>0$. Then

$$
\begin{aligned}
& {\left[\frac{A\left(W_{l}\left(F_{m}+G_{k}\right)^{p}\right)}{B\left(W_{l}\left(\mathscr{F}_{n}+\mathscr{G}_{t}\right)^{r}\right)}\right]^{1 /(p-r)}} \\
& \quad \leq\left[\frac{A\left(W_{l} F_{m}^{p}\right)}{B\left(W_{l} \mathscr{F}_{n}^{r}\right)}\right]^{1 /(p-r)}+\left[\frac{A\left(W_{l} G_{k}^{p}\right)}{B\left(W_{l} \mathscr{G}_{t}^{r}\right)}\right]^{1 /(p-r)} .
\end{aligned}
$$

Proof. By using the Minkowski inequality for positive linear functionals, we get

$$
\begin{aligned}
& A^{1 / p}\left(W_{l}\left(F_{m}+G_{k}\right)^{p}\right) \\
& \quad \leq A^{1 / p}\left(W_{l} F_{m}^{p}\right)+A^{1 / p}\left(W_{l} G_{k}^{p}\right), \\
& B^{1 / r}\left(W_{l}\left(\mathscr{F}_{n}+\mathscr{G}_{t}\right)^{r}\right) \geq B^{1 / r}\left(W_{l} \mathscr{F}_{n}^{r}\right)+B^{1 / r}\left(W_{l} \mathscr{G}_{t}^{r}\right) .
\end{aligned}
$$

By using discrete Hölder's inequality, we get

$$
\begin{gathered}
A^{1 / p}\left(W_{l} F_{m}^{p}\right)+A^{1 / p}\left(W_{l} G_{k}^{p}\right)=\left(\frac{A\left(W_{l} F_{m}^{p}\right)}{B\left(W_{l} \mathscr{F}_{n}^{r}\right)}\right)^{1 / p} \\
\cdot B^{1 / p}\left(W_{l} \mathscr{F}_{n}^{r}\right)+\left(\frac{A\left(W_{l} G_{k}^{p}\right)}{B\left(W_{l} \mathscr{G}_{t}^{r}\right)}\right)^{1 / p} B^{1 / p}\left(W_{l} \mathscr{G}_{t}^{r}\right) \\
\leq\left[\left[\frac{A\left(W_{l} F_{m}^{p}\right)}{B\left(W_{l} \mathscr{F}_{n}^{r}\right)}\right]^{1 /(p-r)}\right.
\end{gathered}
$$




$$
\begin{aligned}
& \left.+\left[\frac{A\left(W_{l} G_{k}^{p}\right)}{B\left(W_{l} \mathscr{G}_{t}^{r}\right)}\right]^{1 /(p-r)}\right]^{(p-r) / p}\left(B^{1 / r}\left(W_{l} \mathscr{F}_{n}^{r}\right)\right. \\
& \left.+B^{1 / r}\left(W_{l} \mathscr{G}_{t}^{r}\right)\right)^{r / p} .
\end{aligned}
$$

Now by combining (23) and (24), we obtain inequality (22).

Theorem 14. Let $W_{l}, F_{m}, G_{k}, \mathscr{F}_{n}, \mathscr{G}_{t}$ be defined as in (20) such that $W_{l}>0$ and $F_{m}, G_{k}, \mathscr{F}_{n}, \mathscr{G}_{t} \geq 0$. Suppose that $A$ and $B$ satisfy $\left(A_{1}\right)$ and $\left(A_{2}\right)$ and

$$
\begin{gathered}
W_{l} F_{m}^{p}, W_{l} G_{k}^{p}, W_{l}\left(F_{m}+G_{k}\right)^{p}, W_{l} \mathscr{F}_{n}^{r}, W_{l} \mathscr{G}_{t}^{r}, \\
W_{l}\left(\mathscr{F}_{n}+\mathscr{G}_{t}\right)^{r} \in L,
\end{gathered}
$$

where $p>2,1>r>0$ such that $p \geq 2(p-r)$ and $B\left(W_{l} \mathscr{F}_{n}^{r}\right), B\left(W_{l} \mathscr{G}_{t}^{r}\right)>0$. Then

$$
\begin{aligned}
& {\left[\frac{A\left(W_{l}\left(F_{m}+G_{k}\right)^{p}\right)}{B\left(W_{l}\left(\mathscr{F}_{n}+\mathscr{G}_{t}\right)^{r}\right)}\right]^{1 /(p-r)}} \\
& \quad \leq\left[\frac{A\left(W_{l} F_{m}^{p}\right)}{B\left(W_{l} \mathscr{F}_{n}^{r}\right)}\right]^{1 /(p-r)}+\left[\frac{A\left(W_{l} G_{k}^{p}\right)}{B\left(W_{l} \mathscr{G}_{t}^{r}\right)}\right]^{1 /(p-r)}-\mathscr{K}
\end{aligned}
$$

$$
\begin{aligned}
& {\left[\frac{A\left(W_{l}\left(F_{m}+G_{k}\right)^{p}\right)}{B\left(W_{l}\left(\mathscr{F}_{n}+\mathscr{G}_{t}\right)^{r}\right)}\right]^{1 /(p-r)}} \\
& \quad \leq\left[\frac{A\left(W_{l} F_{m}^{p}\right)-A\left(W_{l}\left|F_{m}-\left(F_{m}+G_{k}\right)\left(A\left(W_{l} F_{m}\left(F_{m}+G_{k}\right)^{p-1}\right) / A\left(W_{l}\left(F_{m}+G_{k}\right)^{p}\right)\right)\right|^{p}\right)}{B\left(W_{l} \mathscr{F}_{n}^{r}\right)-B\left(W_{l}\left|\mathscr{F}_{n}-\left(\mathscr{F}_{n}+\mathscr{G}_{t}\right)\left(B\left(W_{l} \mathscr{F}_{n}\left(\left(\mathscr{F}_{n}+\mathscr{G}_{t}\right)\right)^{r-1}\right) / B\left(W_{l}\left(\left(\mathscr{F}_{n}+\mathscr{G}_{t}\right)\right)^{r}\right)\right)\right|^{r}\right)}\right]^{1 /(p-r)} \\
& +\left[\frac{A\left(W_{l} G_{k}^{p}\right)-A\left(W_{l}\left|G_{k}-\left(F_{m}+G_{k}\right)\left(A\left(W_{l} G_{k}\left(F_{m}+G_{k}\right)^{p-1}\right) / A\left(W_{l}\left(\left(F_{m}+G_{k}\right)\right)^{p}\right)\right)\right|^{p}\right)}{B\left(W_{l} \mathscr{G}_{t}^{r}\right)-B\left(W_{l}\left|\mathscr{G}_{t}-\left(\mathscr{F}_{n}+\mathscr{G}_{t}\right)\left(B\left(W_{l} \mathscr{G}_{t}\left(\left(\mathscr{F}_{n}+\mathscr{G}_{t}\right)\right)^{r-1}\right) / B\left(W_{l}\left(\left(\mathscr{F}_{n}+\mathscr{G}_{t}\right)\right)^{r}\right)\right)\right|^{r}\right)}\right]^{1 /(p-r)}
\end{aligned}
$$

holds, provided that the denominators of the right-hand side are positive.

Proof. Proof is similar to the proof of Theorem 12.

Next, we obtain the reverse of Theorem 13.

Theorem 16. Let $W_{l}, F_{m}, G_{k}, \mathscr{F}_{n}, \mathscr{G}_{t}$ be defined as in (20) such that $W_{l}>0$ and $F_{m}, G_{k}, \mathscr{F}_{n}, \mathscr{G}_{t} \geq 0$. Suppose that $A$ and $B$ satisfy $\left(A_{1}\right)$ and $\left(A_{2}\right)$ and

$$
\begin{aligned}
& W_{l} F_{m}^{p}, W_{l} G_{k}^{p}, W_{l}\left(F_{m}+G_{k}\right)^{p}, W_{l} \mathscr{F}_{n}^{r}, W_{l} \mathscr{G}_{t}^{r}, \\
& W_{l}\left(\mathscr{F}_{n}+\mathscr{G}_{t}\right)^{r} \in L,
\end{aligned}
$$

holds, where

$$
\begin{aligned}
\mathscr{K} & =\mid\left(\frac{A\left(W_{l} F_{m}^{p}\right)}{B\left(W_{l} \mathscr{F}_{n}^{r}\right)}\right)^{1 / p}-B^{(p-r) / r p}\left(W_{l} \mathscr{F}_{n}^{r}\right) \\
& \left.\cdot \frac{A^{1 / p}\left(W_{l} F_{m}^{p}\right)+A^{1 / p}\left(W_{l} G_{k}^{p}\right)}{B^{1 / r}\left(W_{l} \mathscr{F}_{n}^{r}\right)+B^{1 / r}\left(W_{l} \mathscr{G}_{t}^{r}\right)}\right|^{p /(p-r)} \\
+ & \mid\left(\frac{A\left(W_{l} G_{k}^{p}\right)}{B\left(W_{l} \mathscr{G}_{t}^{r}\right)}\right)^{1 / p}-B^{(p-r) / r p}\left(W_{l} \mathscr{G}_{t}^{r}\right) \\
& \left.\cdot \frac{A^{1 / p}\left(W_{l} F_{m}^{p}\right)+A^{1 / p}\left(W_{l} G_{k}^{p}\right)}{B^{1 / r}\left(W_{l} \mathscr{F}_{n}^{r}\right)+B^{1 / r}\left(W_{l} \mathscr{G}_{t}^{r}\right)}\right|^{p /(p-r)} .
\end{aligned}
$$

Theorem 15. Let $W_{l}, F_{m}, G_{k}, \mathscr{F}_{n}, \mathscr{G}_{t}$ be defined as in (20) such that $W_{l}>0$ and $F_{m}, G_{k}, \mathscr{F}_{n}, \mathscr{G}_{t} \geq 0$. Suppose that $A$ and $B$ satisfy $\left(A_{1}\right)$ and $\left(A_{2}\right)$ and

$$
\begin{gathered}
W_{l} F_{m}^{p}, W_{l} G_{k}^{p}, W_{l}\left(F_{m}+G_{k}\right)^{p}, W_{l} F_{m}\left(F_{m}+G_{k}\right)^{p-1}, \\
W_{l} G_{k}\left(F_{m}+G_{k}\right)^{p-1}, W_{l} \mathscr{F}_{n}^{r}, W_{l} \mathscr{G}_{t}^{r}, W_{l}\left(\mathscr{F}_{n}+\mathscr{G}_{t}\right)^{r}, \\
W_{l} \mathscr{F}_{n}\left(\mathscr{F}_{n}+\mathscr{G}_{t}\right)^{r-1}, W_{l} \mathscr{G}_{t}\left(\mathscr{F}_{n}+\mathscr{G}_{t}\right)^{r-1} \in L,
\end{gathered}
$$

where $p \geq 2,1>r>0$, or $1<r<2$ and $B\left(W_{l} \mathscr{F}_{n}^{r}\right), B\left(W_{l} \mathscr{G}_{t}^{r}\right)>$ 0 . Then
Proof. Proof is similar to the proof of Theorem 11. 


$$
\begin{aligned}
& \geq\left[\frac{\left(A^{1 / p}\left(W_{l} F_{m}^{p}\right)+A^{1 / p}\left(W_{l} G_{k}^{p}\right)\right)^{p}}{\left(B^{1 / r}\left(W_{l} \mathscr{F}_{n}^{r}\right)+B^{1 / r}\left(W_{l} \mathscr{G}_{t}^{r}\right)\right)^{r}}\right]^{1 /(p-r)} \\
& \geq\left(\frac{A\left(W_{l} F_{m}^{p}\right)}{B\left(W_{l} \mathscr{F}_{n}^{r}\right)}\right)^{1 /(p-r)}+\left(\frac{A\left(W_{l} G_{k}^{p}\right)}{B\left(W_{l} \mathscr{G}_{t}^{r}\right)}\right)^{1 /(p-r)} .
\end{aligned}
$$

\section{Generalizations of Bellman's Inequality}

In the following results we get the generalizations of Bellman's inequality, Theorems 4 and 18, respectively.

Theorem 17. Let $W_{l}, F_{m}, G_{k}$ be defined as in (20) such that $W_{l}>0$ and $F_{m}, G_{k} \geq 0$. Suppose that $A$ satisfies $\left(A_{1}\right)$ and $\left(A_{2}\right)$. For $p \in \mathbb{R}$, assume that $W_{l} F_{m}^{p}, W_{l} G_{k}^{p}, W_{l}\left(F_{m}+G_{k}\right)^{p} \in L$. Suppose that $f_{0}, g_{0}>0$ are such that

$$
\begin{aligned}
& f_{0}^{p}-A\left(W_{l} F_{m}^{p}\right)>0, \\
& g_{0}^{p}-A\left(W_{l} G_{k}^{p}\right)>0 .
\end{aligned}
$$

If $p>1$ or $p<0$, then

$$
\begin{aligned}
& \left(\left(f_{0}^{p}-A\left(W_{l} F_{m}^{p}\right)\right)^{1 / p}+\left(g_{0}^{p}-A\left(W_{l} G_{k}^{p}\right)\right)^{1 / p}\right)^{p} \\
& \quad \leq\left(f_{0}+g_{0}\right)^{p}-A\left(W_{l}\left(F_{m}+G_{k}\right)^{p}\right) .
\end{aligned}
$$

If $0<p<1$, then inequality (34) holds in reverse order.

Proof. Let $p>1$. Let $x_{1}, x_{2}, y_{1}, y_{2}$ be nonnegative real numbers. Now from the discrete Minkowski inequality we have

$$
\begin{aligned}
& \left(\left(x_{1}+y_{1}\right)^{p}+\left(x_{2}+y_{2}\right)^{p}\right)^{1 / p} \\
& \quad \leq\left(x_{1}^{p}+x_{2}^{p}\right)^{1 / p}+\left(y_{1}^{p}+y_{2}^{p}\right)^{1 / p} .
\end{aligned}
$$

By applying the substitution

$$
\begin{aligned}
& x_{1}^{p} \longrightarrow f_{0}^{p}-A\left(W_{l} F_{m}^{p}\right), \\
& y_{1}^{p} \longrightarrow g_{0}^{p}-A\left(W_{l} G_{k}^{p}\right), \\
& x_{2}^{p} \longrightarrow A\left(W_{l} F_{m}^{p}\right), \\
& y_{2}^{p} \longrightarrow A\left(W_{l} G_{k}^{p}\right)
\end{aligned}
$$

in (35) and by using Minkowski inequality, we have

$$
\begin{aligned}
& \left(\left(f_{0}^{p}-A\left(W_{l} F_{m}^{p}\right)\right)^{1 / p}+\left(g_{0}^{p}-A\left(W_{l} G_{k}^{p}\right)\right)^{1 / p}\right)^{p} \\
& \quad \leq\left(f_{0}+g_{0}\right)^{p}-\left(A^{1 / p}\left(W_{l} F_{m}^{p}\right)+A^{1 / p}\left(W_{l} G_{k}^{p}\right)\right)^{p} \\
& \quad \leq\left(f_{0}+g_{0}\right)^{p}-A\left(W_{l}\left(F_{m}+G_{k}\right)^{p}\right) .
\end{aligned}
$$

If $p<0$, then (35) holds in reverse order. Now by using the negativity of $p$, we get

$$
\begin{aligned}
& \left(x_{1}+y_{1}\right)^{p}+\left(x_{2}+y_{2}\right)^{p} \\
& \quad \leq\left(\left(x_{1}^{p}+x_{2}^{p}\right)^{1 / p}+\left(y_{1}^{p}+y_{2}^{p}\right)^{1 / p}\right)^{p} .
\end{aligned}
$$

The remaining proof is similar to the case for $p>1$, except that here we apply the substitutions from (36) in (38).

If $0<p<1$, then the reversed inequality in (34) can be proved in a similar way. In this case, (35) holds in reverse order.

Theorem 18. Let $W_{l}, F_{m}, G_{k}$ be defined as in (20) such that $W_{l}>0$ and $F_{m}, G_{k} \geq 0$. Suppose that $A$ satisfies $\left(A_{1}\right)$ and $\left(A_{2}\right)$. For $p \geq 2$, assume that

$$
\begin{gathered}
W_{l} F_{m}^{p}, W_{l} G_{k}^{p}, W_{l}\left(F_{m}+G_{k}\right)^{p}, W_{l} F_{m}\left(F_{m}+G_{k}\right)^{p-1}, \\
W_{l} G_{k}\left(F_{m}+G_{k}\right)^{p-1} \in L
\end{gathered}
$$

and $A\left(W_{l}\left(F_{m}+G_{k}\right)^{p}\right)>0$. Suppose that $f_{0}, g_{0}>0$ are such that

$$
\begin{aligned}
& f_{0}^{p}-A\left(W_{l} F_{m}^{p}\right)>0, \\
& g_{0}^{p}-A\left(W_{l} G_{k}^{p}\right)>0 .
\end{aligned}
$$

Then the following inequality holds:

$$
\begin{aligned}
& \left(\left(f_{0}^{p}-A\left(W_{l} F_{m}^{p}\right)\right)^{1 / p}+\left(g_{0}^{p}-A\left(W_{l} G_{k}^{p}\right)\right)^{1 / p}\right)^{p} \leq\left[\left(f_{0}^{p}\right.\right. \\
& \left.-A\left(W_{l}\left|F_{m}-\left(F_{m}+G_{k}\right) \frac{A\left(W_{l} F_{m}\left(F_{m}+G_{k}\right)^{p-1}\right)}{A\left(W_{l}\left(F_{m}+G_{k}\right)^{p}\right)}\right|^{p}\right)\right)^{1 / p} \\
& +\left(g_{0}^{p}\right. \\
& \left.\left.-A\left(W_{l}\left|G_{k}-\left(F_{m}+G_{k}\right) \frac{A\left(W_{l} G_{k}\left(F_{m}+G_{k}\right)^{p-1}\right)}{A\left(W_{l}\left(F_{m}+G_{k}\right)^{p}\right)}\right|^{p}\right)\right)^{1 / p}\right]^{p} \\
& -A\left(W_{l}\left(F_{m}+G_{k}\right)^{p}\right) .
\end{aligned}
$$

Proof. The proof is similar to the proof of Theorem 17; only here we apply the substitution

$$
\begin{aligned}
& x_{1}^{p} \longrightarrow f_{0}^{p}-A\left(W_{l} F_{m}^{p}\right), \\
& y_{1}^{p} \longrightarrow g_{0}^{p}-A\left(W_{l} G_{k}^{p}\right), \\
& x_{2}^{p} \longrightarrow A\left(W_{l} F_{m}^{p}\right)-A\left(W_{l} \mid F_{m}\right. \\
&\left.-\left.\left(F_{m}+G_{k}\right) \frac{A\left(W_{l} F_{m}\left(F_{m}+G_{k}\right)^{p-1}\right)}{A\left(W_{l}\left(F_{m}+G_{k}\right)^{p}\right)}\right|^{p}\right),
\end{aligned}
$$




$$
\begin{aligned}
& y_{2}^{p} \rightarrow A\left(W_{l} G_{k}^{p}\right)-A\left(W_{l} \mid G_{k}\right. \\
& \left.-\left.\left(F_{m}+G_{k}\right) \frac{A\left(W_{l} G_{k}\left(F_{m}+G_{k}\right)^{p-1}\right)}{A\left(W_{l}\left(F_{m}+G_{k}\right)^{p}\right)}\right|^{p}\right)
\end{aligned}
$$

in (35) and then apply Theorem 8.

\section{Applications on Time Scales Integrals}

A time scale $\mathbb{T}$ is an arbitrary closed subset of $\mathbb{R}$. Time scales calculus provides the unification and extension of discrete and continuous analysis. It is useful for the simultaneous study of discrete and continuous data. For example, when $\mathbb{T}=\mathbb{R}$, the time scale integral is an ordinary integral, and when $\mathbb{T}=\mathbb{Z}$, the time scale integral becomes a sum. Here, we give the definition and results for diamond- $\alpha$ integral, but all the results of this section also hold for other time scales integrals, by using the fact that time scales integrals satisfy the properties of positive linear functionals. For a detailed introduction of time scales integral, we refer to [9-13]. Dresher's and Bellman's inequalities for time scales integrals are given in [6].

Definition 19 (diamond- $\alpha$ integral [13, Definition 3.2]). Let $f: \mathbb{T} \rightarrow \mathbb{R}$ be continuous function and let $a, b \in \mathbb{T}$. Then the diamond- $\alpha$ integral of $f$ from $a$ to $b$ is defined by

$$
\begin{aligned}
\int_{a}^{b} f(t) \diamond_{\alpha} t=\alpha \int_{a}^{b} f(t) \Delta t+(1-\alpha) \int_{a}^{b} f(t) \nabla t & \\
& 0 \leq \alpha \leq 1 .
\end{aligned}
$$

Remark 20. From the above definition it is clear that for $\alpha=1$ the diamond- $\alpha$ integral reduces to the standard delta integral and for $\alpha=0$ the diamond- $\alpha$ integral reduces to the standard nabla integral.

Moreover, if $\mathbb{T}=\mathbb{R}$, then

$$
\int_{a}^{b} f(t) \diamond_{\alpha} t=\int_{a}^{b} f(t) \mathrm{d} t
$$

if $\mathbb{T}=\mathbb{Z}$, then

$$
\int_{a}^{b} f(t) \diamond_{\alpha} t=\alpha \sum_{t=a}^{b-1} f(t)+(1-\alpha) \sum_{t=a+1}^{b} f(t)
$$

if $\mathbb{T}=h \mathbb{Z}$, where $h>0$, then

$$
\begin{aligned}
& \int_{a}^{b} f(t) \diamond_{\alpha} t \\
& \quad=h\left(\alpha \sum_{k=a / h}^{b / h-1} f(k h)+(1-\alpha) \sum_{k=a / h+1}^{b / h} f(k h)\right) ;
\end{aligned}
$$

if $\mathbb{T}=q^{\mathbb{N}_{0}}$, where $q>1$, then

$$
\begin{gathered}
\int_{a}^{b} f(t) \diamond_{\alpha} t=(q-1)\left(\alpha \sum_{k=\log _{q}(a)}^{\log _{q}(b)-1} q^{k} f\left(q^{k}\right)+(1-\alpha)\right. \\
\left.\quad \sum_{k=\log _{q}(a)+1}^{\log _{q}(b)} q^{k-1} f\left(q^{k}\right)\right) .
\end{gathered}
$$

The following results are immediate consequence of the results obtained in Sections 2, 3, and 4, respectively.

Corollary 21. Let $a, b \in \mathbb{T}$ with $a<b$. Suppose that $w, f_{i}, g_{i} \in$ $\mathrm{C}\left([a, b]_{\mathbb{T}},[0, \infty)\right)$. If $p>2,1>r>0$ such that $p \geq 2(p-r)$ and $\int_{a}^{b} w(t) g_{i}^{r}(t) \diamond_{\alpha} t>0$ for $1 \leq i \leq n$, then

$$
\begin{aligned}
& {\left[\frac{\int_{a}^{b} w(t)\left(\sum_{i=1}^{n} f_{i}(t)\right)^{p} \diamond_{\alpha} t}{\int_{a}^{b} w(t)\left(\sum_{i=1}^{n} g_{i}(t)\right)^{r} \diamond_{\alpha} t}\right]^{1 /(p-r)}} \\
& \quad \leq \sum_{i=1}^{n}\left[\frac{\int_{a}^{b} w(t) f_{i}^{p}(t) \diamond_{\alpha} t}{\int_{a}^{b} w(t) g_{i}^{r}(t) \diamond_{\alpha} t}\right]^{1 /(p-r)}-K,
\end{aligned}
$$

where

$$
\begin{aligned}
K= & \sum_{i=1}^{n} \mid\left(\frac{\int_{a}^{b} w(t) f_{i}^{p}(t) \diamond_{\alpha} t}{\int_{a}^{b} w(t) g_{i}^{r}(t) \diamond_{\alpha} t}\right)^{1 / p} \\
& -\left(\int_{a}^{b} w(t) g_{i}^{r}(t) \diamond_{\alpha} t\right)^{(p-r) / r p} \\
& \left.\cdot \frac{\sum_{i=1}^{n}\left(\int_{a}^{b} w(t) f_{i}^{p}(t) \diamond_{\alpha} t\right)^{1 / p}}{\sum_{i=1}^{n}\left(\int_{a}^{b} w(t) g_{i}^{r}(t) \diamond_{\alpha} t\right)^{1 / r}}\right|^{p /(p-r)} .
\end{aligned}
$$

Corollary 22. Let $a, b \in \mathbb{T}$ with $a<b$. Suppose that $w, f_{i}, g_{i} \in$ $\mathrm{C}\left([a, b]_{\mathbb{T}},[0, \infty)\right)$. If $p \geq 2,1>r>0$ or $1<r<2$ and $\int_{a}^{b} w(t) g_{i}^{r}(t) \diamond_{\alpha} t>0$ for $1 \leq i \leq n$, then

$$
\begin{aligned}
& {\left[\frac{\int_{a}^{b} w(t)\left(\sum_{i=1}^{n} f_{i}(t)\right)^{p} \diamond_{\alpha} t}{\int_{a}^{b} w(t)\left(\sum_{i=1}^{n} g_{i}(t)\right)^{r} \diamond_{\alpha} t}\right]^{1 /(p-r)}} \\
& \leq \sum_{i=1}^{n}\left[\frac{\int_{a}^{b} w(t) f_{i}^{p}(t) \diamond_{\alpha} t-\int_{a}^{b} w(t) U_{i}(t) \diamond_{\alpha} t}{\int_{a}^{b} w(t) g_{i}^{r}(t) \diamond_{\alpha} t-\int_{a}^{b} w(t) V_{i}(t) \diamond_{\alpha} t}\right]^{1 /(p-r)}
\end{aligned}
$$


holds, provided that the denominator of the right-hand side is positive, where

$$
\begin{aligned}
& U_{i}(t)=\mid f_{i}(t) \\
& -\sum_{i=1}^{n} f_{i}(t) \frac{\int_{a}^{b} w(t) f_{i}(t)\left(\sum_{i=1}^{n} f_{i}(t)\right)^{p-1} \nabla_{\alpha} t}{\int_{a}^{b} w(t)\left(\sum_{i=1}^{n} f_{i}(t)\right)^{p} \diamond_{\alpha} t} \mid, \\
& V_{i}(t)=\mid g_{i}(t) \\
& -\left.\sum_{i=1}^{n} g_{i}(t) \frac{\int_{a}^{b} w(t) g_{i}(t)\left(\sum_{i=1}^{n} g_{i}(t)\right)^{r-1} \diamond_{\alpha} t}{\int_{a}^{b} w(t)\left(\sum_{i=1}^{n} g_{i}(t)\right)^{r} \diamond_{\alpha} t}\right|^{r} .
\end{aligned}
$$

Corollary 23. Let $a, b \in \mathbb{T}$ with $a<b$. Suppose that $\left\{w_{i}(t)\right\}_{i=1}^{l}$, $\left\{f_{i}(t)\right\}_{i=1}^{m},\left\{g_{i}(t)\right\}_{i=1}^{k} \in \mathrm{C}\left([a, b]_{\mathbb{T}},[0, \infty)\right)$, and $W_{l}, F_{m}, G_{k}$ defined as in (20) are such that $W_{l}>0$ and $F_{m}, G_{k} \geq 0$. If $p \geq 1>r>0$ and $\int_{a}^{b} W_{l}(t) F_{m}^{r}(t) \diamond_{\alpha} t, \int_{a}^{b} W_{l}(t) G_{k}^{r}(t) \diamond_{\alpha} t>0$, then

$$
\begin{gathered}
\left(\frac{\int_{a}^{b} W_{l}(t)\left(F_{m}(t)+G_{k}(t)\right)^{p} \diamond_{\alpha} t}{\int_{a}^{b} W_{l}(t)\left(F_{m}(t)+G_{k}(t)\right)^{r} \diamond_{\alpha} t}\right)^{1 /(p-r)} \\
\leq\left(\frac{\int_{a}^{b} W_{l}(t) F_{m}^{p}(t) \diamond_{\alpha} t}{\int_{a}^{b} W_{l}(t) F_{m}^{r}(t) \diamond_{\alpha} t}\right)^{1 /(p-r)} \\
+\left(\frac{\int_{a}^{b} W_{l}(t) G_{k}^{p}(t) \diamond_{\alpha} t}{\int_{a}^{b} W_{l}(t) G_{k}^{r}(t) \nabla_{\alpha} t}\right)^{1 /(p-r)}
\end{gathered}
$$

holds, where

$$
\begin{aligned}
& W_{l}(t)=W_{l}\left(w_{1}(t), w_{2}(t), \ldots, w_{l}(t)\right), \\
& F_{m}(t)=F_{m}\left(f_{1}(t), f_{2}(t), \ldots, f_{m}(t)\right), \\
& G_{k}(t)=G_{k}\left(g_{1}(t), g_{2}(t), \ldots, g_{k}(t)\right) .
\end{aligned}
$$

Corollary 24. Let $W_{l}, F_{m}, G_{k}$ satisfy the conditions of Corollary 23 with $p>2,1>r>0$ and $p \geq 2(p-r)$. Then

$$
\begin{gathered}
\left(\frac{\int_{a}^{b} W_{l}(t)\left(F_{m}(t)+G_{k}(t)\right)^{p} \diamond_{\alpha} t}{\int_{a}^{b} W_{l}(t)\left(F_{m}(t)+G_{k}(t)\right)^{r} \diamond_{\alpha} t}\right)^{1 /(p-r)} \\
\leq\left(\frac{\int_{a}^{b} W_{l}(t) F_{m}^{p}(t) \diamond_{\alpha} t}{\int_{a}^{b} W_{l}(t) F_{m}^{r}(t) \diamond_{\alpha} t}\right)^{1 /(p-r)} \\
+\left(\frac{\int_{a}^{b} W_{l}(t) G_{k}^{p}(t) \diamond_{\alpha} t}{\int_{a}^{b} W_{l}(t) G_{k}^{r}(t) \diamond_{\alpha} t}\right)^{1 /(p-r)}-\mathscr{R}
\end{gathered}
$$

holds, where

$$
\begin{aligned}
\mathscr{R} & =\left(\mid\left(\frac{\int_{a}^{b} W_{l}(t) F_{m}^{p}(t) \diamond_{\alpha} t}{\int_{a}^{b} W_{l}(t) F_{m}^{r}(t) \diamond_{\alpha} t}\right)^{1 / p}\right. \\
& -\left.\left(\int_{a}^{b} W_{l}(t) F_{m}^{r}(t) \diamond_{\alpha} t\right)^{(p-r) / r p} \delta\right|^{p /(p-r)} \\
& \left.+\left.\left|\left(\frac{\int_{a}^{b} W_{l}(t) G_{k}^{p}(t) \diamond_{\alpha} t}{\int_{a}^{b} W_{l}(t) G_{k}^{r}(t) \diamond_{\alpha} t}\right)^{1 / p}\right|^{(p-r) / r p}\right|^{p /(p-r)}\right) \\
& \left.-\left(\int_{a}^{b} W_{l}(t) G_{k}^{r}(t) \diamond_{\alpha} t\right)^{(1)}\right)
\end{aligned}
$$

such that

$\delta$

$$
=\frac{\left(\int_{a}^{b} W_{l}(t) F_{m}^{p}(t) \diamond_{\alpha} t\right)^{1 / p}+\left(\int_{a}^{b} W_{l}(t) G_{k}^{p}(t) \diamond_{\alpha} t\right)^{1 / p}}{\left(\int_{a}^{b} W_{l}(t) F_{m}^{r}(t) \diamond_{\alpha} t\right)^{1 / r}+\left(\int_{a}^{b} W_{l}(t) G_{k}^{r}(t) \diamond_{\alpha} t\right)^{1 / r}} .
$$

Corollary 25. Let $W_{l}, F_{m}, G_{k}$ satisfy the conditions of Corollary 23 with $p \geq 2,1>r>0$, or $1<r<2$. Then

$$
\begin{aligned}
& \left(\frac{\int_{a}^{b} W_{l}(t)\left(F_{m}(t)+G_{k}(t)\right)^{p} \diamond_{\alpha} t}{\int_{a}^{b} W_{l}(t)\left(F_{m}(t)+G_{k}(t)\right)^{r} \diamond_{\alpha} t}\right)^{1 /(p-r)} \\
& \quad \leq\left(\frac{\int_{a}^{b} W_{l}(t) F_{m}^{p}(t) \diamond_{\alpha} t-\int_{a}^{b} W_{l}(t) \mathscr{U}(t) \diamond_{\alpha} t}{\int_{a}^{b} W_{l}(t) F_{m}^{r}(t) \diamond_{\alpha} t-\int_{a}^{b} W_{l}(t) \mathscr{V}(t) \diamond_{\alpha} t}\right)^{1 /(p-r)} \\
& +\left(\frac{\int_{a}^{b} W_{l}(t) G_{k}^{p}(t) \diamond_{\alpha} t-\int_{a}^{b} W_{l}(t) \mathscr{W}(t) \diamond_{\alpha} t}{\int_{a}^{b} W_{l}(t) G_{k}^{r}(t) \diamond_{\alpha} t-\int_{a}^{b} W_{l}(t) \mathscr{T}(t) \diamond_{\alpha} t}\right)^{1 /(p-r)}
\end{aligned}
$$

holds, provided that the denominators on the right-hand side are positive, where

$$
\begin{aligned}
& \mathscr{U}(t)=\mid F_{m}(t)-\left(F_{m}(t)+G_{k}(t)\right) \\
& \cdot \frac{\int_{a}^{b} W_{l}(t) F_{m}(t)\left(F_{m}(t)+G_{k}(t)\right)^{p-1} \nabla_{\alpha} t}{\int_{a}^{b} W_{l}(t)\left(F_{m}(t)+G_{k}(t)\right)^{p} \diamond_{\alpha} t} \mid, \\
& \mathscr{V}(t)=\mid F_{m}(t)-\left(F_{m}(t)+G_{k}(t)\right) \\
& \left.\cdot \frac{\int_{a}^{b} W_{l}(t) F_{m}(t)\left(F_{m}(t)+G_{k}(t)\right)^{r-1} \diamond_{\alpha} t}{\int_{a}^{b} W_{l}(t)\left(F_{m}(t)+G_{k}(t)\right)^{r} \diamond_{\alpha} t}\right|^{r},
\end{aligned}
$$




$$
\begin{gathered}
\mathscr{W}(t)=\mid G_{k}(t)-\left(F_{m}(t)+G_{k}(t)\right) \\
\cdot \frac{\int_{a}^{b} W_{l}(t) G_{k}(t)\left(F_{m}(t)+G_{k}(t)\right)^{p-1} \nabla_{\alpha} t}{\int_{a}^{b} W_{l}(t)\left(F_{m}(t)+G_{k}(t)\right)^{p} \diamond_{\alpha} t} \mid, \\
\mathscr{T}(t)=\mid G_{k}(t)-\left(F_{m}(t)+G_{k}(t)\right) \\
.\left.\frac{\int_{a}^{b} W_{l}(t) G_{k}(t)\left(F_{m}(t)+G_{k}(t)\right)^{r-1} \diamond_{\alpha} t}{\int_{a}^{b} W_{l}(t)\left(F_{m}(t)+G_{k}(t)\right)^{r} \diamond_{\alpha} t}\right|^{r} .
\end{gathered}
$$

Corollary 26. Let $W_{l}, F_{m}, G_{k}$ satisfy the conditions of Corollary 23 with $p \leq 0<r<1$. Then

$$
\begin{gathered}
\left(\frac{\int_{a}^{b} W_{l}(t)\left(F_{m}(t)+G_{k}(t)\right)^{p} \diamond_{\alpha} t}{\int_{a}^{b} W_{l}(t)\left(F_{m}(t)+G_{k}(t)\right)^{r} \diamond_{\alpha} t}\right)^{1 /(p-r)} \\
\geq\left(\frac{\int_{a}^{b} W_{l}(t) F_{m}^{p}(t) \diamond_{\alpha} t}{\int_{a}^{b} W_{l}(t) F_{m}^{r}(t) \diamond_{\alpha} t}\right)^{1 /(p-r)} \\
+\left(\frac{\int_{a}^{b} W_{l}(t) G_{k}^{p}(t) \diamond_{\alpha} t}{\int_{a}^{b} W_{l}(t) G_{k}^{r}(t) \diamond_{\alpha} t}\right)^{1 /(p-r)}
\end{gathered}
$$

Corollary 27. Let $W_{l}, F_{m}, G_{k}$ satisfy the conditions of Corollary 23. Suppose that $f_{0}, g_{0}>0$ are such that

$$
\begin{aligned}
& f_{0}^{p}-\int_{a}^{b} W_{l}(t) F_{m}^{p}(t) \diamond_{\alpha} t>0, \\
& g_{0}^{p}-\int_{a}^{b} W_{l}(t) G_{k}^{p}(t) \diamond_{\alpha} t>0 .
\end{aligned}
$$

If $p>1$ or $p<0$, then

$$
\begin{aligned}
& \left(\left(f_{0}^{p}-\int_{a}^{b} W_{l}(t) F_{m}^{p}(t) \diamond_{\alpha} t\right)^{1 / p}\right. \\
& \left.+\left(g_{0}^{p}-\int_{a}^{b} W_{l}(t) G_{k}^{p}(t) \diamond_{\alpha} t\right)^{1 / p}\right)^{p} \leq\left(f_{0}+g_{0}\right)^{p} \\
& -\int_{a}^{b} W_{l}(t)\left(F_{m}(t)+G_{k}(t)\right)^{p} \diamond_{\alpha} t .
\end{aligned}
$$

If $0<p<1$, then the above inequality holds in reverse order.
Corollary 28. Let $W_{l}, F_{m}, G_{k}$ satisfy the conditions of Corollary 23 with $p \geq 2$. Suppose that $\int_{a}^{b} W_{l}(t)\left(F_{m}(t)+G_{k}(t)\right)^{p} \diamond_{\alpha} t>$ 0 and $f_{0}, g_{0}>0$ are such that

$$
\begin{aligned}
& f_{0}^{p}-\int_{a}^{b} W_{l}(t) F_{m}^{p}(t) \diamond_{\alpha} t>0, \\
& g_{0}^{p}-\int_{a}^{b} W_{l}(t) G_{k}^{p}(t) \diamond_{\alpha} t>0 .
\end{aligned}
$$

Then the following inequality holds:

$$
\begin{aligned}
& \left(\left(f_{0}^{p}-A\left(W_{l} F_{m}^{p}\right)\right)^{1 / p}+\left(g_{0}^{p}-A\left(W_{l} G_{k}^{p}\right)\right)^{1 / p}\right)^{p} \leq\left[\left(f_{0}^{p}\right.\right. \\
& \left.-A\left(W_{l}\left|F_{m}-\left(F_{m}+G_{k}\right) \frac{A\left(W_{l} F_{m}\left(F_{m}+G_{k}\right)^{p-1}\right)}{A\left(W_{l}\left(F_{m}+G_{k}\right)^{p}\right)}\right|^{p}\right)\right)^{1 / p} \\
& +\left(g_{0}^{p}\right. \\
& \left.\left.-A\left(W_{l}\left|G_{k}-\left(F_{m}+G_{k}\right) \frac{A\left(W_{l} G_{k}\left(F_{m}+G_{k}\right)^{p-1}\right)}{A\left(W_{l}\left(F_{m}+G_{k}\right)^{p}\right)}\right|^{p}\right)\right)^{1 / p}\right]^{p} \\
& -A\left(W_{l}\left(F_{m}+G_{k}\right)^{p}\right) .
\end{aligned}
$$

\section{Conflict of Interests}

The authors declare that there is no conflict of interests regarding the publication of this paper.

\section{References}

[1] P. S. Bullen, Handbook of Means and their Inequalities, Kluwer Academic Publishers, London, UK, 2003.

[2] M. Dresher, "Moment spaces and inequalities," Duke Mathematical Journal, vol. 20, pp. 261-271, 1953.

[3] D. S. Mitrinović, Analytic Inequalities, (In Cooperation with P. M. Vasić), Springer, Berlin, Germany, 1970.

[4] D. S. Mitrinović, J. E. Pečarić, and A. M. Fink, Classical and New Inequalities in Analysis, Kluwer Academic, Dodrecht, The Netherlands, 1993.

[5] J. E. Pečarić, F. Proschan, and Y. L. Tong, Convex Functions, Partial Orderings, and Statistical Applications, vol. 187 of Mathematics in Science and Engineering, Academic Press, Boston, Mass, USA, 1992.

[6] M. Anwar, R. Bibi, M. Bohner, and J. Pečarić, "Integral inequalities on time scales via the theory of isotonic linear functionals," Abstract and Applied Analysis, vol. 2011, Article ID 483595, 16 pages, 2011.

[7] R. Bibi, "Some improvements of the Popoviciu, Bellman and Diaz-Metcalf inequalities via superquadratic functions," Mathematical Inequalities \& Applications, vol. 18, no. 3, pp. 981-989, 2015.

[8] S. Banić and S. Varošanec, "Functional inequalities for superquadratic functions," International Journal of Pure and Applied Mathematics, vol. 43, no. 4, pp. 537-549, 2008. 
[9] M. Bohner and G. S. Guseinov, "Multiple integration on time scales," Dynamic Systems and Applications, vol. 14, no. 3-4, pp. 579-606, 2005.

[10] M. Bohner and G. S. Guseinov, "Multiple Lebesgue integration on time scales," Advances in Difference Equations, vol. 2006, Article ID 26391, 12 pages, 2006.

[11] M. Bohner and A. Peterson, Dynamic Equations on Time Scales: An Introduction with Applications, Birkhäuser, Boston, Mass, USA, 2001.

[12] M. Bohner and A. Peterson, Advances in Dynamic Equations on Time Scales, Birkhäuser, Boston, Mass, USA, 2003.

[13] Q. Sheng, M. Fadag, J. Henderson, and J. M. Davis, "An exploration of combined dynamic derivatives on time scales and their applications," Nonlinear Analysis. Real World Applications, vol. 7, no. 3, pp. 395-413, 2006. 


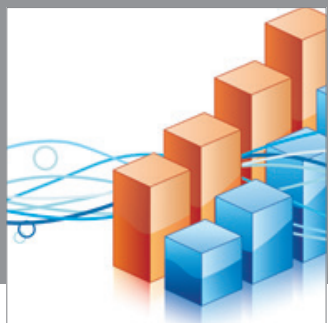

Advances in

Operations Research

mansans

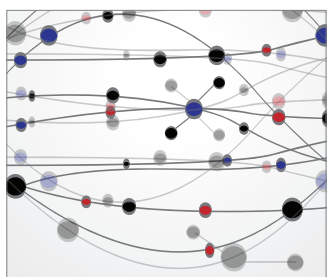

The Scientific World Journal
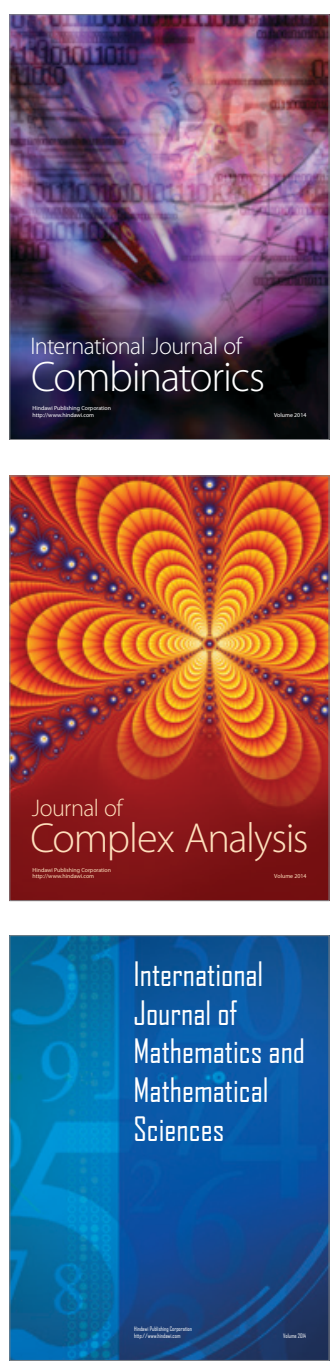
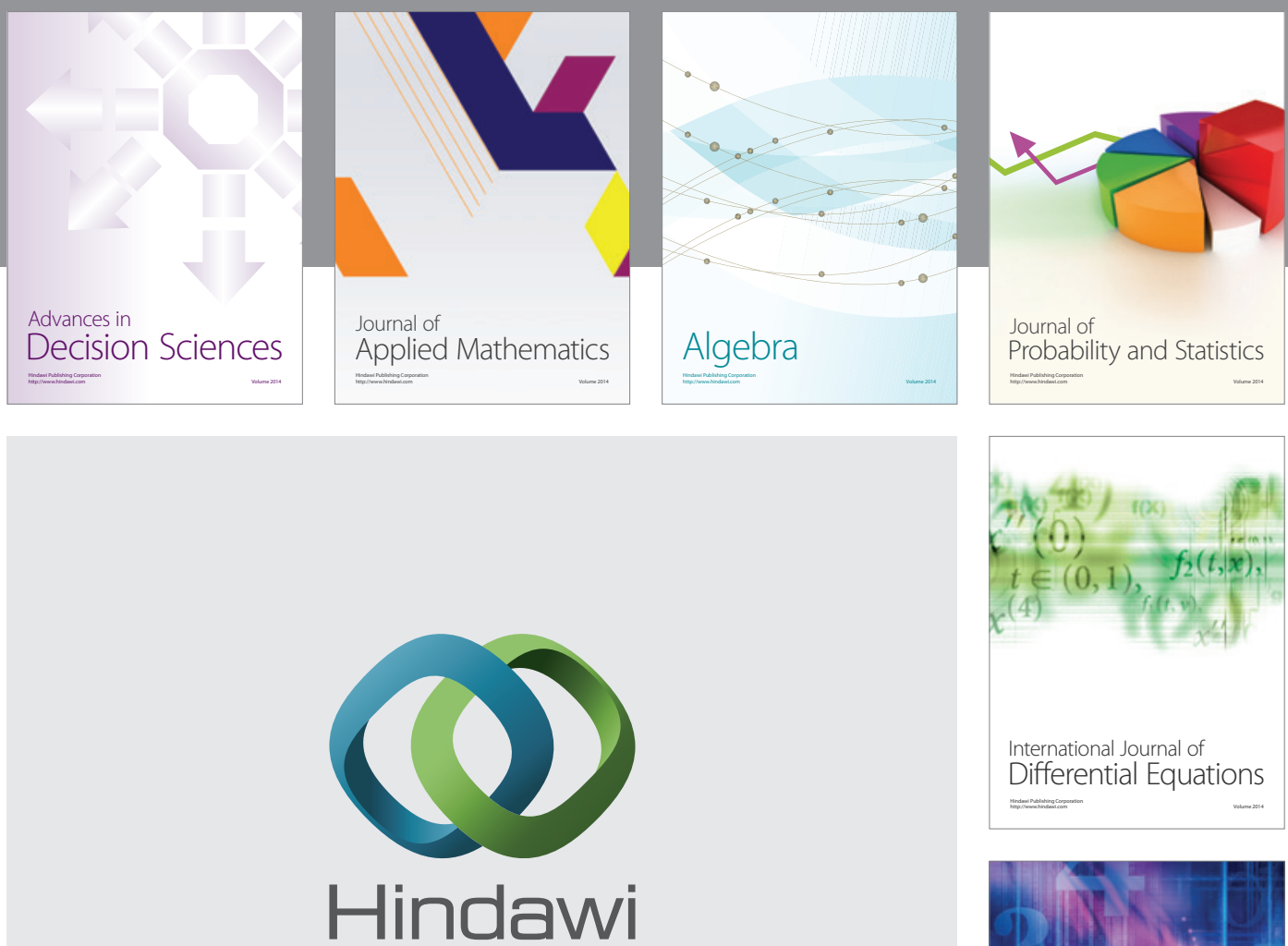

Submit your manuscripts at http://www.hindawi.com
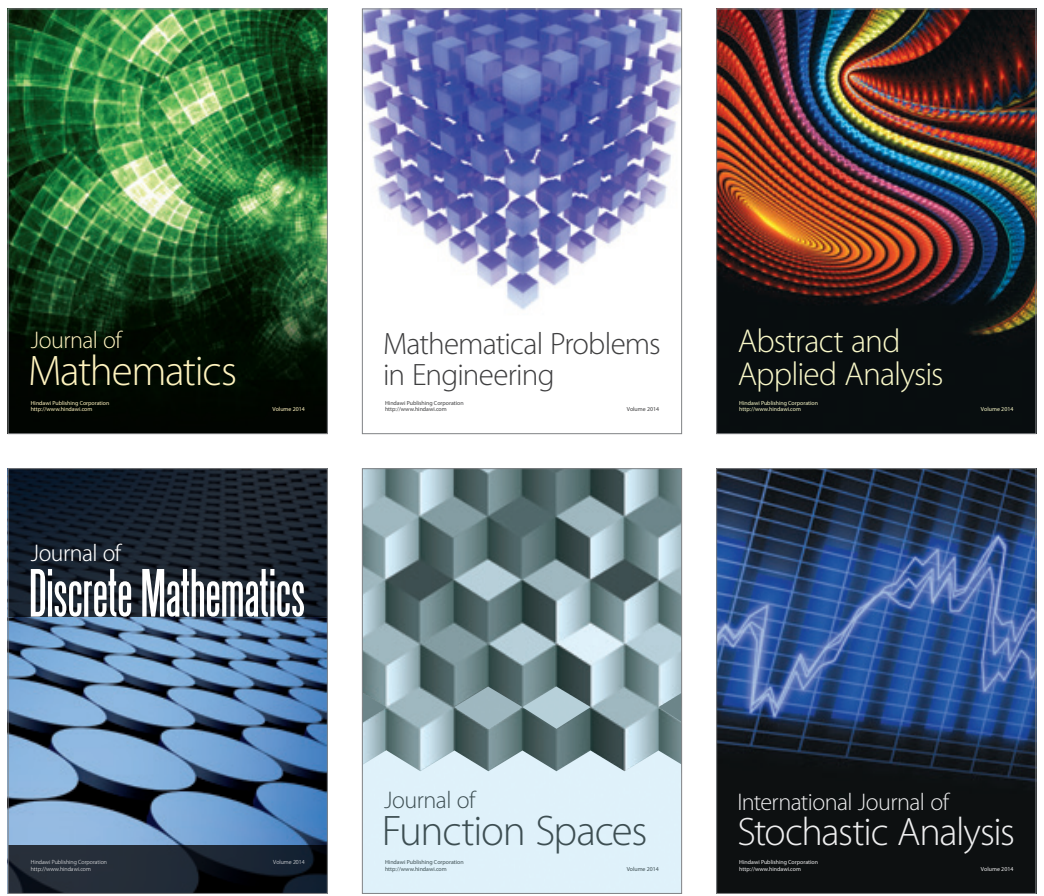

Journal of

Function Spaces

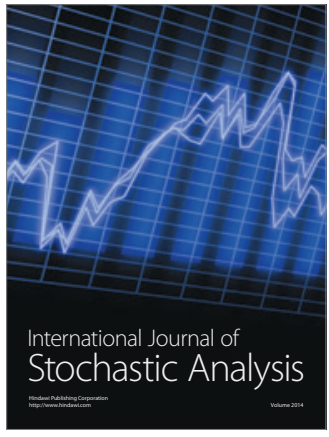

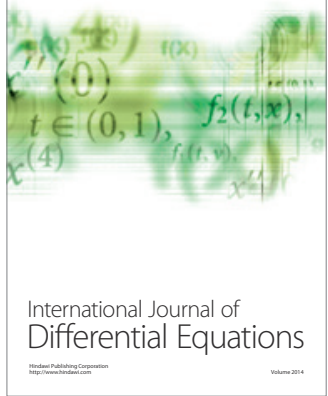
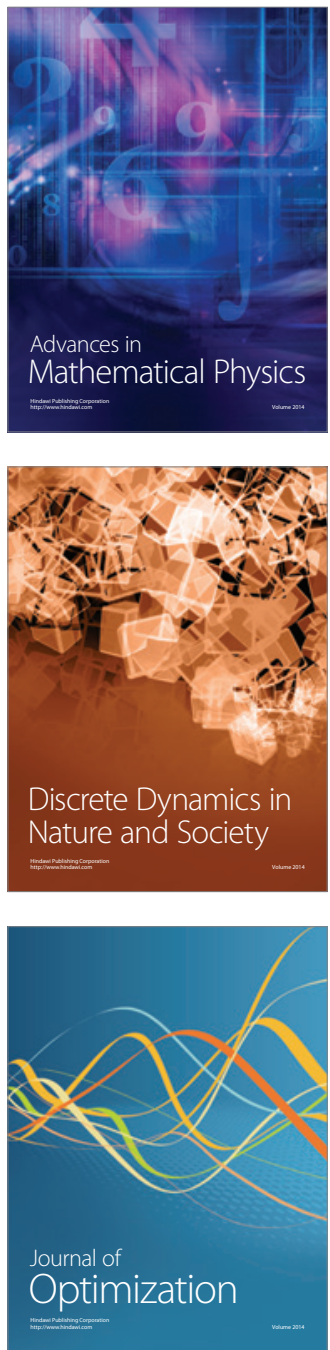